\title{
Introducing an Approach for Secure Authentication using Iris Recognition
}

\author{
Charvi Gahlot \\ Student \\ GDGWI, Gurgaon, Haryana
}

\author{
Deepak Sharma \\ Lecturer \\ GDGWI, Gurgaon, Haryana
}

\begin{abstract}
This paper proposes an approach to iris recognition system for securely authenticating user. Eye images are captured using a USB webcam and are enhanced. Segmentation is done using an algorithm based on hough transform . Then the selected portion of image is converted to polar coordinates. Distinctive features of the iris are extracted using Haar wavelet transform and encoded. Iris binary codes after filteration will be stored in system database \& hamming distance calculation is done for comparing two codes.
\end{abstract}

\section{Keywords}

Iris, image processing, image encoding, biometric, security

\section{INTRODUCTION}

A biometric identification system is one which identifies a person automatically based on his unique characteristics. Biometrics are used for access control.

Iris recognition - Each individual has a unique \& random iris pattern. A person's iris is identified by analyzing distinct features like freckles, rings, crypts, ridges \& furrows present in the iris pattern. But, the size of the iris is small to scan from a large distance. So try to keep the distance between the user \& camera minimum for accurately extracting the iris features.

Iris recognition is capable of having numerous applications in fields of business security, criminal investigation, employee identification, ATM, etc. Iris recognition is preferred for authentication because of its high accuracy.

\section{LITERATURE SURVEY}

Currently deployed iris recognition systems are highly efficient, fast and easy to use and produce no false matches even in several million comparison tests [1]. Iris recognition can easily be considered as the most reliable form of biometric technology [2].

It is important to use a camera with the correct amount of illumination. As iris is a planar object, its image will be insensitive to the angle of illumination.

An iris recognition process usually has the following stages: Image Acquisition, segmentation, normalization, feature extraction, encoding and matching.

The Daugman system used an LED based point light source along with a camera while acquiring an image. Subtle infrared illumination is used to reduce reflection from the convex cornea.

The segmentation stage is critical to the success of an iris recognition system [3] . Many researchers have assumed that the pupil \& iris circumferences are concentric.
For segmentation , Daugman proposed an integro-differential operator. While, Wildes used circular hough transforms to detect the iris and pupil contours. Parabolic hough transform was also used to detect the upper $\&$ lower eyelids.

Kong and Zhang proposed a method to localize eyelashes using 1D Gabor filters.

The test results published so far have usually been produced using carefully imaged irises under favourable conditions.[3] Normalization is done to compensate for inconsistencies between eye images mainly due to pupil dilation .

The wavelet transform of a function is its decomposition into a set of functions which form a base and are called the 'wavelets'. Research groups like Ma et al, Tisse et al, Zhu et al and Wildes et al have used Haar wavelets for feature extraction. Haar wavelet transformation performs consecutive iterations to achieve the level of detail required. For iris recognition , 5 iterations are needed [4].

Daugman used the Hamming distance as the matching metric for iris recognition. In large-scale applications, a database containing hundreds of iris codes is maintained \& comparison is done with each of them.

In 2008, Hidalgo, Garcia and Lorenzo created an iris recognition software in java using the JAVAVis Library.

\section{PROPOSED WORK}

\subsection{Image Capture}

The proposed iris recognition application will capture an image of the user's eye using a webcam. Connect a USB webcam to your computer and bring it close to your eye (left or right) while acquiring an image. Take care of the lighting conditions in the surrounding environment. The image should capture the rich details of iris patterns.

The image will be filtered to remove noise. Its brightness and contrast will also be adjusted before processing.

\subsection{Image Processing}

Locate in an image the outer boundary of the iris \& the pupil. The pupil \& iris circumferences are not assumed to be concentric.

Binarization of the image is done to emphasize the pupil region for better localization. Edge detection is done using sobel operator. For segmentation, an algorithm based on hough transform will be used which tolerates bright spots of illumination inside the pupil.

\subsubsection{Hough Transform}

Hough transform is a standard method which can help us determine the parameters of simple geometric shapes like circles present in a scanned image. 
A circle is defined using 3 parameters: radius (r) \& centre coordinates $(\mathrm{c}, \mathrm{d})$.

Equation of circle is given by formula 1 below:

$$
(x-c)^{2}+(y-d)^{2}=r^{2} \quad \text {.........[formula 1] }
$$

There is mapping among the hough space (x, y) \& the space of parameters $(c, d, r)$. A maximum point in the hough space corresponds to the radius \& centre coordinates of the iris best defined by the edge points [3].

\subsubsection{Normalization}

Separate the iris from the rest of the image and make it dimensionally consistent. This is called Normalization. The circular iris image will be transformed into a rectangular image. This is demonstrated by figure 1 .

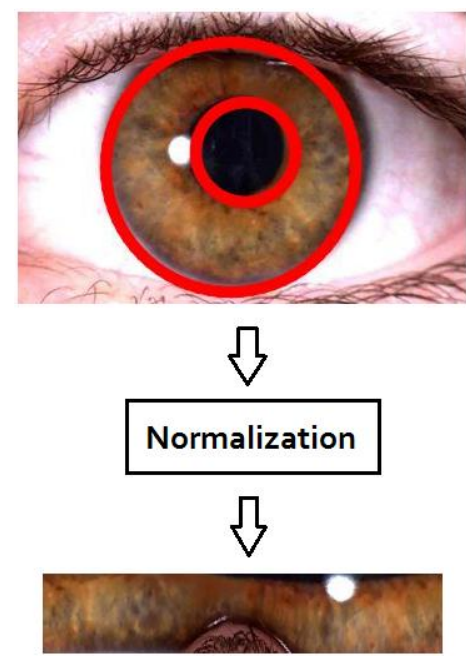

Fig 1 : Normalization

The input image need not be a gray image. Eyelids \& eyelashes must also be localized and secluded from the normalized image.

\subsection{Image Encoding}

Next, useful information needs to be extracted from the normalized iris image. This information is then encoded into a binary template. Using Haar wavelet transform, a template is created by a band pass decomposition of the iris image. The image is broken down into 4 sub-images :

- an image that has been high pass filtered in the horizontal and vertical directions

- an image that has been low pass filtered in the vertical and high pass filtered in the horizontal direction

- an image that has been low pass filtered in both directions

- an image that has been low pass filtered in the horizontal and vertical directions

Five iterations of this process are sufficient to obtain the distinctive information about human iris required to uniquely identify a person. The output of this process is some coefficients in the form of a vector. These real values are converted to binary zeroes $\&$ ones.

A database will be maintained which is a collection of iris templates. To authenticate a user, his binary template is compared with each of the templates stored in database. In case of a perfect match, the user is granted access.

\subsection{Matching}

Two iris templates/codes are matched for similarity using certain matching metrics. One such metric is the calculation of a Hamming distance. To compare the templates X \& Y, the Hamming distance is defined as the sum of dissimilar bits (sum of X xor Y) over the total number of bits in the template $(\mathrm{N})$, as shown by formula 2 .

$$
H D=\frac{1}{N} \sum_{i=1}^{N}\left(X_{i} \text { xor } Y_{i}\right) \quad \text {......[formula 2] }
$$

Hamming distance between 2 templates of the same iris should be close to 0.0 .

\section{SYSTEM ARCHITECTURE}

It is proposed that this application will perform two functions:

- $\quad$ enroll new user - block diagram shown in figure 2

- $\quad$ authenticate user - block diagram shown in figure 3

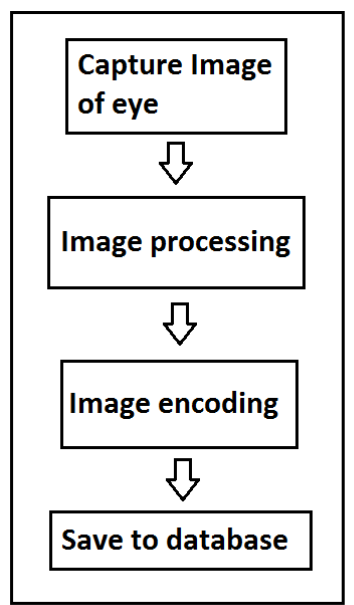

Fig 2 : Block diagram for enrolling new user

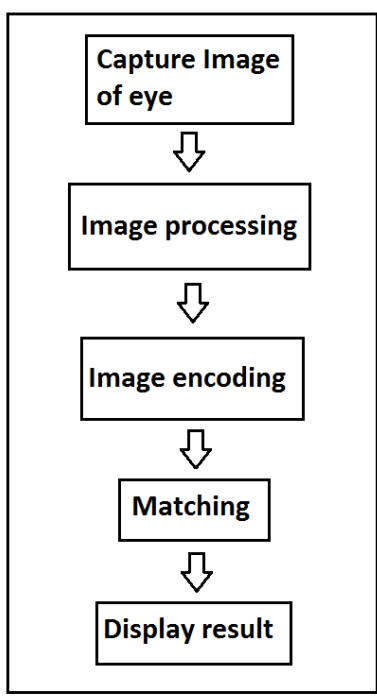

Fig 3 : Block diagram for user authentication 


\section{CONCLUSIONS}

Iris recognition is an emerging biometric technique for personal authentication. It is reliable \& allows no false acceptance. An iris recognition system must tackle problems of noisy images, blur images, reflections \& low contrast.

After analysis of the current technologies which can be used to implement such a software, java technology has been finalized for the development of this project.

Table 1. A comparison of development platforms

\begin{tabular}{|l|l|l|l|l|l|}
\hline & JAVA & MATLAB & C++ & VB.NET & PYTHON \\
\hline $\begin{array}{l}\text { CROSS- } \\
\text { PLATFORM }\end{array}$ & $\checkmark$ & $\checkmark$ & $\times$ & $\times$ & $\checkmark$ \\
\hline OPEN SOURCE & $\checkmark$ & $\times$ & $\checkmark$ & $\checkmark$ & $\checkmark$ \\
\hline $\begin{array}{l}\text { ANDROID } \\
\text { APPLICATION } \\
\text { S }\end{array}$ & $\checkmark$ & $\times$ & $\checkmark$ & $\times$ & $\times$ \\
\hline
\end{tabular}

Some image processing libraries of java may come handy. People are mostly familiar with java \& usually have it preinstalled on their systems. This software can be enhanced in future to develop an android application for iris recognition so that mobile/tablet security can be improved.

\section{REFERENCES}

[1] John Daugman, "How Iris Recognition Works," IEEE transaction on circuits and systems for video technology, vol. 14, no. 1, pp. 1-3, january 2004.
[2] S. Sanderson and J. Erbetta, "Authentication for secure environments based on iris scanning technology," IEEE Colloquium on Visual Biometrics, 2000.

[3] L. Masek, "Recognition of Human Iris Patterns for Biometric Identification,""M.Thesis2003.

[4] V.Larrumbe Hidalgo, L.Martin García and M.Taboada Lorenzo , "Iris recognition using the JAVAVis Library," International Journal of Interactive Multimedia and Artificial Intelligence, Vol. 1, No 1, ISSN 1989-1660.

[5] Manoj K. Vairalkar, "Edge Detection of Images Using Sobel Operator," International Journal of Emerging Technology and Advanced Engineering, vol. 2, issue 1, january 2012.

[6] Meherchilakalapudi, "KNOW ABOUT - BIOMETRIC AUTHENTICATION SYSTEM," May 30,2009 , available:

http://meherchilakalapudi.wordpress.com/2009/05/30/kn ow-about-biometric-authentication-system/

[7] Jafar M. H. Ali and Aboul Ella Hassanien ,"An Iris Recognition System to Enhance E-security Environment Based on Wavelet Theory," AMO - Advanced Modeling and Optimization, Volume 5, Number 2, 2003.

[8] Nandan.T.Murthy ,"Technical Seminar On IRIS RECOGNITION," city engineering college.

[9] Roger F. Larico Chavez, Yuzo Iano, Member IEEE, and Vicente B. Sablón, "Process of Recognition of Human Iris: Fast Segmentation of the Iris," University of Campinas, available: http://www.decom.fee.unicamp.br/ rlarico/iris/localizati oniris.pdf 